Methods: We extracted population-based longitudinally linked administrative health data for patients 16 years or older with a first diagnostic code of 711.xx (ICD9-CM) and M00.xx (ICD10-AM) in WA in the period 1990-2010. Annual incidence rates (IR), risk factors during 14.5 years lookback and outcomes including standardized mortality rates (SMR) during 10.1 years follow-up are reported. Results: A total of 2,777 patients $(67 \%$ male, mean age $49.8 \pm 20.5)$ received a first diagnostic code for PyA. The AIR increased from 4.5 to $11.8 / 100,000$ over time as did age at onset ( 45.1 to 55.4 years) and proportion of female patients (23 to $36 \%$ ). There was no seasonal variation in PyA incidence but a higher rate of predisposing comorbidities in female patients. Knees (33.6\%) and hands $(22 \%)$ were most frequently affected with $28.4 \%$ of positive cultures not due to $\mathrm{Gr}+$ cocci. Mean hospital stay was 8 days, 30-day readmittance and mortality rate was $12.8 \%$ and $3.1 \%$ respectively. During ten years follow-up serious infections $(43 \%)$, new diagnosis of osteoarthrosis $(20 \%)$, joint replacement $(10.8 \%)$, osteomyelitis (6\%), and crystal arthropathy $(6.3 \%)$ were the most common morbidities. SMR were increased across all age and gender categories (Table) but highest in females aged 16-40 (SMR 25.9)

Table 1. Mortality rates (MR) per 1000 person years in patients with pyogenic arthritis compared with age (at death) and gender matched categories from the general population by standardised mortality rate (SMR)

\begin{tabular}{lcccccc}
\hline Gender & Age & Deaths & Person years & MR PyA & MR Gen pop * & SMR \\
\hline Male & & & & & & \\
& $16-40$ & 27 & 4015 & 6.72 & 0.892 & 7.53 \\
& $40-59$ & 80 & 7106 & 11.25 & 2.972 & 3.78 \\
& $>60$ & 331 & 7366 & 44.93 & 21.55 & 2.08 \\
Female & All & $\mathbf{4 3 8}$ & $\mathbf{1 8 4 8 7}$ & $\mathbf{2 3 . 6 9}$ & $\mathbf{5 . 8 2 0}$ & $\mathbf{4 . 0 7}$ \\
& $16-40$ & 11 & 1026 & 10.72 & 0.41 & 25.95 \\
& $40-59$ & 40 & 2769 & 14.44 & 1.75 & 8.21 \\
& $>60$ & 208 & 4088 & 50.88 & 24.20 & 2.10 \\
& All & $\mathbf{2 5 9}$ & $\mathbf{7 8 8 3}$ & $\mathbf{3 2 . 8 5}$ & $\mathbf{5 . 5 0}$ & $\mathbf{5 . 9 6}$ \\
\hline
\end{tabular}

Conclusion: The incidence of PyA has increased significantly between 1990 and 2010 in WA. PyA associates with a 3\% in-hospital mortality rate and significant late bone and joint morbidity including osteomyelitis. PyA associated with excess mortality across age and gender categories, most markedly in younger female patients. REFERENCES:

[1] Ross JJ. Septic arthritis of native joints. Infect Dis Clin North Am 2017;31:203-18

Acknowledgements: The authors would like to acknowledge the support of the Western Australian Data Linkage Branch, the Western Australian Department of Health, and the data custodians of, the Hospital and Morbidity Data Collection, the Emergency Department Data Collection the WA Cancer Register and the WA Death Register for their assistance with the study

Disclosure of Interests: None declared

DOI: 10.1136/annrheumdis-2021-eular.1344

\section{OP0096 EXPOSURE TO DENGUE INFECTION DO NOT RAISE RISK OF RHEUMATOID ARTHRITIS: FINDINGS FROM THE MALAYSIAN EPIDEMIOLOGICAL INVESTIGATION OF RHEUMATOID ARTHRITIS (MYEIRA) CASE- CONTROL STUDY}

L. K. $\operatorname{Tan}^{1,2}$, C. L. Too ${ }^{1,10}$, A. F. Nurul-Aain ${ }^{1}$, A. A. Siti-Aisyah¹, S. Wahinuddin ${ }^{2}$, A. Osman ${ }^{2}$, I. S. Lau ${ }^{3}$, H. Hussein ${ }^{4}$, N. S. Shahril', S. C. Gun ${ }^{5}$, E. Mageswaren ${ }^{4}$, M. S. Mohamed Said ${ }^{6}$, A. Mohd. Mokhtar ${ }^{7}$, R. Azmillah ${ }^{3}$, M. Othman ${ }^{8}$, L. Alfredsson ${ }^{9}$, L. Klareskog ${ }^{10}$, M. Shahnaz ${ }^{11}$, L. Padyukov ${ }^{10} .{ }^{1}$ Allergy and Immunology Research Center, Institute for Medical Research, Ministry of Health Malaysia, Selangor, Malaysia; ${ }^{2}$ Faculty of Medicine, Universiti Kuala Lumpur Royal College of Medicine Perak, Perak, Malaysia; ${ }^{3}$ Department of Medicine, Selayang Hospital, Selangor, Malaysia; ${ }^{4}$ Department of Medicine, Putrajaya Hospital, Putrajaya, Malaysia; ${ }^{5}$ Department of Medicine, Tuanku Ja'afar Hospital, Negeri Sembilan, Malaysia; ${ }^{6}$ Faculty of Medicine, University Kebangsaan Malaysia Medical Center, Kuala Lumpur, Malaysia; ${ }^{7}$ Department of Medicine, Tengku Ampuan Afzan Hospital, Pahang, Malaysia; ${ }^{8}$ Department of Medicine, Raja Permaisuri Bainun Hospital, Perak, Malaysia; ${ }^{9}$ Institute of Environmental Medicine, Karolinska Institutet, Stockholm, Malaysia; ${ }^{10}$ Department of Medicine, Rheumatology Unit, Karolinska Institutet and Karolinska University Hospital, Stockholm, Sweden; ${ }^{11}$ Ministry of Health Malaysia, Federal Government Administrative Center, Putrajaya, Malaysia

Background: Dengue infection is associated with joints pain mimicking disease onset symptom of rheumatoid arthritis (RA). However, there is lack of epidemiological studies on exposure to dengue infection and risk of future RA.

Objectives: We investigated the relationship between exposure to dengue infection and risk of developing different subsets of RA, defined by the presence of anti-citrullinated peptide antibody (ACPA) in the multi-ethnic Malaysian population.

Methods: Serum samples from 1,235 RA cases (i.e. 516 Malay, 254 Chinese 405 Indians and 60 others/mixed-ethnicity) and 1,624 epidemiological matched population-based controls (i.e. 1,023 Malay, 208 Chinese, 297 Indians and 96 others/mixed-ethnicity) were assayed for presence of dengue $\lg G$ antibody using World Health Organization recommended ELISA kits. Positive results of dengue IgG antibodies indicates previous exposure to dengue infection(s). We performed chi-square and Mann-Whitney $U$ analysis to determine the association of ever-exposed dengue infection with ACPA-positive/ACPA-negative RA and to investigate the antibody frequency and levels among the studied populations.

Results: We observed high occurrence of dengue IgG antibody in the overal RA cases $(79.7 \%)$ and matched controls $(77.3 \%)$, with no significant differences detected between the ACPA subsets of RA. Ethnicity stratification analysis revealed a decrease risk of developing ACPA-positive RA in the Indian patients with positive dengue $\operatorname{lgG}$ antibody ( $\mathrm{OR}=0.59,95 \% \mathrm{Cl}=0.37-0.94, \mathrm{p}=0.03$ ), and in particular patients with elevated level of dengue IgG antibody (OR $=0.44$ $95 \% \mathrm{Cl}=0.25-0.78, \mathrm{p}<0.05)$. On the other hand, the significant decrease mean levels of dengue IgG antibody were observed in the ACPA-positive RA subset for all three major ethnic groups (i.e. Malay, $p<0.0001$, Chinese, $p<0.01$ and Indian<0.05) (Figure 1). No association was observed between presence of dengue IgG antibody and ACPA-negative RA subset.

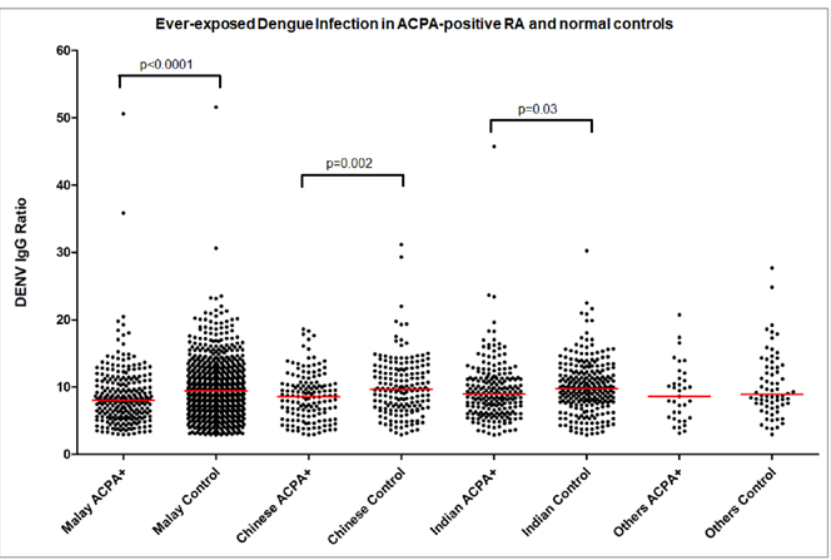

Figure 1. Comparison of mean dengue IgG antibody level between ever-exposed dengue infection RA cases, stratified by ACPA status. Comparison of median dengue IgG antibody level between the ever-exposed dengue infection ACPA-positive RA and normal controls in the four ethnic groups. The red line indicates the mean level of dengue $\lg G$ antibody level

Conclusion: Our findings demonstrated that exposure to dengue infection do not increase the risk of developing future RA in the multi-ethnic Malaysian population. The inverse associations observed in the Indian ethnic group are in line with the other studies investigating exposure to viral infection and risk of RA. REFERENCES:

[1] Sherina et al (2017) Low levels of antibodies against common viruses associate with anti-citrullinated protein antibody-positive rheumatoid arthritis; implications for disease aetiology. Arthritis Research \& Therapy 2017, 19:2169

[2] Gissel García et. al. (2011) Long-term persistence of clinical symptoms in dengue-infected persons and its association with immunological disorders. International Journal of Infectious Diseases 15 (2011) e38-e43

Acknowledgements: The authors would like to thank the Director General of Health, Ministry of Health Malaysia for supporting this study. The authors are also indebted to participants for their kind participation. This study was financially supported by the Ministry of Health, Malaysia (JPP-IMR 17-025) and the short-term research grant by UniKL RCMP (str16037).

Disclosure of Interests: None declared

DOI: 10.1136/annrheumdis-2021-eular.1684

OP0097
LISTERIA MONOCYTOGENES. DESCRIPTION AND
ANALYSIS OF CASES IN AN IMMUNODEPRESSED
POPULATION BY RHEUMATIC DISEASES

M. Lisbona Muñoz ${ }^{1}$, P. León ${ }^{1}$, G. Lopez Antequera ${ }^{1}$, E. Rubio-Romero' ${ }^{1}$ H.U.Virgen del Rocío, Rheumatology, Seville, Spain

Background: Listeria monocytogenes is a gram-positive bacteria that cause the invasive disease listeriosis. Human clinical syndromes are infrequent, mostly appearing in immunosuppressed individuals, newborns, the elderly, pregnant women, and occasionally healthy patients. 